\title{
EVALUATION OF SURFACE ROUGHNESS AND MICROBIAL BIOFILM ADHESION OF DIFFERENT ORTHODONTIC ARCH-WIRES
}

\author{
Marwa Ali Tawfik* ${ }^{*}$ Tayseer Maaly** and Rasha Mokhtar EL-Nagar***
}

\begin{abstract}
Objectives: To assess the capability of different orthodontic arch-wires to retain oral biofilm and to correlate the adhesion to surface roughness of the wires.

Methods: Four types of orthodontic arch-wires were used for the study, Nickel-titanium (NiTi), Copper nickel-titanium (Cu-NiTi), Beta Titanium (TMA ) \& Beta III Titanium (CNA) new arch wires and 4 weeks after intraoral usage, were examined for Surface roughness (SR) using an atomic force microscope (AFM). Adhesion of Streptococcus mutans (MS), Staphylococcus aureus (SA), and Candida albicans (CA) were performed using colony count method. Statistically, the following tests were done: analysis of variance, Pearson correlation coefficient test, post hoc Tukey test.
\end{abstract}

Results: The four wire types showed significant increases in $\mathrm{SR}(\mathrm{P}<0.05)$ after 4 weeks of intra-oral usage, TMA wires recorded the highest roughness values while the lowest ones were for NiTi wires. Bacterial adhesion was detected on all wires, ANOVA showed significant differences between the wires concerning MS, SA and CA adhesion. A significant positive correlation ( $\mathrm{P}=.001)$ was observed between bacterial adhesion and surface roughness after intra-oral exposure.

Conclusions: SR of the wires increased after intra-oral use and there was a positive correlation with the biofilm adhesion.

KEY WORDS: Biofilm adhesion, surface roughness, orthodontic arch-wires.

\section{INTRODUCTION}

Orthodontics involves the use of a range of corrective devices, such as braces and plates, to encourage the teeth to be aligned properly. However, appliances used in orthodontic treatment probably produce negative effects on oral hygiene. The site where the tooth surface contacting the brackets is liable to accumulation of oral bacteria leading to biofilm adhesion. Oral biofilm, or "dental plaque", is hard to be eliminated and continuous teeth brushing is not always enough to eliminate the plaque from

* Lecturer of Orthodontic, Faculty of Dentistry, Mansoura University.

** Lecturer of Dental Biomaterials, Faculty of Dentistry, Zagazig University.

*** Lecturer of Medical Microbiology and Immunology, Faculty of Medicine, Mansoura University. 
the adhesion sites, like the bracket-adhesive-tooth surface areas, and the zone between brackets and the free gingiva. ${ }^{1}$

The Streptococci, as mentioned in many researches, has an important role in the promoting of the primary stages of biofilm creation in the oral cavity. Streptococcus bacteria in conjunction with other bacterial types are claimed to be the main colonizers of dental biofilm development 2. It was found that the role of Streptococcus species inside the oral cavity is smaller in comparison to that of Staphylococcus one, due to carnivore food regime is less fermentable type and their salivary $\mathrm{pH}$ degree is mostly alkaline. ${ }^{3}$

Candida albicans (C. Albicans) is the main fungus colonies which was isolated during different oral infectious diseases, and many studies have shown that different oral bacteria can adhere to $\mathrm{C}$. Albicans in oral biofilm infections and can alter its pathogenicity. ${ }^{4}$ At the same time, existence of $\mathrm{C}$. Albicans can change the behavior of the bacterial microbiota. 5 Streptococcus mutans, the main bacteria responsible for early childhood caries (ECC), which can be accumulated with C. albicans in a bi-directional interaction complex ${ }^{6}$

As known in last few years, the most common orthodontic arch-wire used is the Nickel titanium (Ni-Ti) alloys. Recently, Copper Nickel-Titanium (Cu-Ni-Ti) arch wire, an alloy composed of copper, nickel, titanium, and chromium, was introduced to improve the different tooth movements in certain cases . ${ }^{7}$ It was reported that a copper metallic surface has antimicrobial property and that viruses, bacteria and yeasts are soon killed on it. ${ }^{8}$

Another type of orthodontic arch wires was introduced by Charles Burstone in the 1980's which is The Beta Titanium (TMA). TMA is a stabilized titanium alloy in the beta phase formed of titanium $(79 \%)$, molybdenum (11\%), zirconium (6\%), and tin (4\%). As a result, its modulus of elasticity is lower with more spring back property than that of the steel type, also it provides a combination of great shape memory, suitable enough stiffness, better formability, weldability and high degree of attrition. All these characteristics give rise of a TMA type of wire which has both characters stiffness and elasticity, which offer ideal properties for the finishing stage in orthodontic treatment. ${ }^{9}$ Beta-III Titanium Nickel-free alloy arch wires called CNA, it is a replacement for TMA arch wires. The wire exhibits excellent ductility for insertion of loops and bends minimizing important chairside time. Previous studies ${ }^{10,11}$ concluded that CNA wires presented the greatest flexibility between stainless steel, nitinol and TMA alloys.

Bacterial adhesion is affected by the surface properties of biomaterials, especially surface roughness. A rough surface provides opportunities for bacterial attachment by expanding the surface area leading to more adhesion areas and preventing the displacement of bacterial colonies. ${ }^{12,13}$

The aim of this study was to evaluate the surface roughness and microbial biofilm adhesion of four different orthodontic arch-wires: Nitinol (Ni-Ti), Copper-Nitinol (Cu-Ni-Ti ), Beta-Titanium (TMA) and Beta-III Titanium (CNA) .

\section{MATERIALS AND METHODS}

Four types of an orthodontic arch wires (Ni-Ti, $\mathrm{Cu}-\mathrm{Ni}$-Ti, TMA and CNA) were used in the study (Table 1).

\section{Sample size calculation}

The calculated sample size of the study was 7 wires for each group at $5 \%$ level of significance and $80 \%$ power of the study, using G Power 3 sample size calculator. It was found in previous study (Mahasen et al) that mean surface roughness for type 1 wire $=1.1$ with SD 0.31 and mean surface roughness of type 2 wire $=1.6$ with SD 0.38 . The sample size was increased to 10 wires for each group to compensate for protocol failures with incomplete data to increase the study power. 
TABLE (1) Orthodontic arch wires used in the study.

\begin{tabular}{|c|c|c|c|}
\hline Group number & Number of patients & Cross section size & Composition \\
\hline 1 & 10 & $0.017 " x 0.025 "$ & Copper -Nickel titanium alloy" (Cu-Ni-Ti) \\
\hline 2 & 10 & $0.017 " x$ 0.025" & Nickel-Titanium alloy $^{* *}(\mathrm{Ni}-\mathrm{Ti})$ \\
\hline 3 & 10 & $0.017 ” x$ 0.025” & Titanium Nickel-free alloy ${ }^{* * * *}$ (CAN) \\
\hline 4 & 10 & $0.017 ”$ x $0.025 ”$ & Beta Titanium Molybdenum ${ }^{* * * * * *}$ alloy (TMA) \\
\hline
\end{tabular}

Forty orthodontic patients (22 female and 18 male) were elected from the clinic of Orthodontic Department (Faculty of Dentistry, Mansoura University, Egypt). All patients had permanent dentition, age range of $15.13 \pm 3.25$, with moderate to severe crowding with good oral hygiene and healthy normal gingiva, and the absence of any systematic or dental abnormal condition. They did not have any previous medication for a month prior to or during the orthodontic.

The patients or their parents signed an informed consent which includes information about the treatment approach, this study was accepted by the Ethics Committee of Mansoura University.

After an orthodontic diagnostic records and the treatment plan was established for all the participating patients, the treatment procedure was accomplished according to the following protocol to guard against the effect of the bracket on the results, the selected patients had straight wire, the same brackets of 0.022 " slot Roth system and molar tubes* were used, they were bonded using orthodontic composite $^{* *}$ according to the manufacture instructions. A series of orthodontic arch wires were used for each patient starting from size 0.012 " till reaching 0.016 " 0.022 " according to the treatment progress of each case. The elastomeric ties were used during all the treatment steps. After this stage of orthodontic treatment, patients were divided randomly into 4 equal groups according to table (1).

Before engaging the new wire in the brackets 2 $\mathrm{cm}$ were cut form the distal end of each wire as received, cleaned by distilled water, dried by air jet oil free, packed and identified to be ready for the surface roughness analysis by the atomic force microscope.

After engaging the rectangular 0.017 " $\mathrm{x} 0.025$ " orthodontic wire for the patients in the four different groups 4 weeks were calculated for each participant.

For the used arch wire and before its removal from the patient's mouth the area opposite to the right and left premolars were marked by a permanent marker to ensure that, all the piece of wires which were examined concerning the examination of the biofilm formation or the surface roughness analysis, were subjected to the same oral environment. Then the wires were sectioned from the midline by a sharp cutter to achieve two halves of the wire for the forty patients.

The right half of the arch wire was gripped carefully to guard against any iatrogenic biofilm removal, rinsed with an air/water spray to get rid of the food debris, then they were air dried, the marked

* OrthOrganizers, Carlsbad, calif.,USA.

** Grengloo, Ormco, Orange, Calif, USA. 
piece of wire was cut, packed into a sterilizing plastic bag. All the bags were identified with the patient's name, the date of insertion and removal and the number of the study group. The collected wires were sent to the clinical laboratory at the same day to analysis of the biofilm formation. The other left half of the wire was cleaned, rinsed with physiologic saline, dried with an oil free air syringe, sterilized, the premolar marked parts were sectioned by a sharp cutter, backed separately and identified like the other right section to be ready for the atomic force analysis.

\section{Bacterial Attachment on Orthodontic Wire:}

Quantitative analysis of the microbial biofilm formation was performed by viable bacterial cell counting.(CA),(SA),(MS), were cultured separately in an Eppendorf tube which was containing 1.5 $\mathrm{mL}$ of brain heart infusion (BHI) medium, where the piece of wire under examination were soaked in. After a period of forty hours of incubation at 37 degree Celsius under aerobic conditions, each wire segment was washed 2 times in sterile phosphatebuffered saline (PBS) and then, then they were transferred to second sterile Eppendorf tube.

\section{For viable cell counting:}

Each piece of orthodontic used arch wire was sonicated using ultrasonic vibration in $1 \mathrm{~mL}$ PBS. The PBS was diluted in a serial manner and each $100 \mathrm{~mL}$ was spread on top of a BHI agar plate. After 2-3 days (incubation period), bacterial colonies were analyzed and counted from each sample, and finally the calculation of the relative colony forming units was done.

\section{Quantitative Biofilm Measurement:}

All the collected samples were washed very carefully with PBS to eliminate the non- adherent bacteria. Then $0.5 \%$ crystal violet solution was used to stain the sample for thirty minutes, and washed 5 times with distilled water, and all the samples was left to dry at room temperature for another $30 \mathrm{~min}$ utes. After that, $95 \%$ ethanol was added to solubilize the crystal violet. The absorbance was established at $595 \mathrm{~nm}$ using an enzyme-linked immunosorbent assay (ELISA) reader. The strains were considered to be a significant biofilm former when the OD595 threshold value was over 0.5 .

\section{Surface Roughness}

All the $2 \mathrm{~cm}$ piece of the "as received" arch wire which were cut before insertion in the oral cavity, and the collected "used" left prepared sterilized sections were scanned to have a pre and post surface roughness values for each type of the different arch wire used in this study. This was measured by atomic force microscopy ((Nanosurf FlexAFM 3, Gräubernstrasse 12, 4410 Liestal, Switzerland). The AFM was operated in contact mode using nonconductive silicon nitride probe using Nano surf C3000 (version 3.5.0.31) software with the following specifications: scan area, $10 \times 10 \mu \mathrm{m}^{2}$ and number of data points: 256 x 256 line; at scan rate $1 \mathrm{HZ}$.

Three different areas were assessed, measuring 5 x $5 \mathrm{~mm}$ each, one in the center of the piece of wire, at other two readings , $2 \mathrm{~mm}$ on the right and left direction, all measurements were on the labial surface of the wire segments, which were fixed on a glass slide before examination and insertion in the AFM. Finally, the average value SR ( $\mathrm{Ra}$ ) was evaluated.

\section{Statistical Analysis}

All the collected data was analyzed using Statistical Package for Social Science software computer program version 23 (SPSS, Inc., Chicago, IL, USA). Data were presented in mean and standard

\footnotetext{
* Ormco, Orange, Calif, USA.

** 3M unitek, Saint paul, MN, USA.

*** Ortho Organizers, Carlsbad, Calif., USA.

**** Ormco, Orange, Calif, USA.
} 
deviation. One-way Analysis of variance (ANOVA) and Tuckey were used for comparing data. Pearson's correlation coefficient was used to correlate between surface roughness \& Microbial biofilm adhesion test for MS, SA and CA. $P$ value less than 0.05 was considered statistically significant.

\section{RESULTS}

\section{Microbial Biofilm Adhesion}

The adherence of MS, SA, CA to the wires is shown in Table (2). For SA and MS adhesion, NiTi wire showed the lowest mean values $(0.174 \pm 0.055$, $0.192 \pm 0.035$, respectively), while CuNiTi showed the lowest value for CA $(0.110 \pm 0.016)$. The highest values for MS, SA, and CA adhesion were recorded for TMA wire $(0.376 \pm 0.14,0.549 \pm 0.17$ and $0.462 \pm 0.054$, respectively). ANOVA analysis demonstrated a significant difference between the different types of wires for MS, SA, and CA $(\mathrm{P}<0.05)$. Tukey's test revealed that TMA wire had significantly higher MS and CA adhesion than the other three types, while higher SA adhesion than NiTI and CuNiTi wires, with no significant difference with CNA. There is no significant difference between CuNiTi and NiTi wires in respect to SA and MS ahesion, while they significantly different for CA adhesion. CNA wire showed significantly higher MS, SA and CA adhesion than NiTi wire, while significantly higher CA adhesion only with CuNiTi.

\section{Surface Roughness}

Surface roughness values of new and used wires are shown in Table 3. All of the archwires studied increased in surface roughness after intraoral exposure. For all wires, a significant difference of SR was found between new and used wires $(\mathrm{P}<0.05)$. The highest mean values of SR were recorded for the new and used TMA wires (165.4 \pm 13.45 , $217.4 \pm 12.59$ respectively) whereas the lowest values were for the new and used NiTi wires $(54.15 \pm 9.722$ and $88.61 \pm 9.957$ respectively) followed by new and used $\mathrm{Cu}$ NiTi wires $(63.84 \pm 5.452$ and $107.3 \pm 8.489$ respectively) and new and used CNA $(74.24 \pm 5.322$ and 147.5 \pm 8.886 respectively). ANOVA analysis showed significant difference in surface roughness between the four types of examined arch wires either new or used $(\mathrm{P}<0.05)$. The results of the Tukey's test revealed a significant difference between TMA wires and the other three types, and there is no significant difference between NiTi and $\mathrm{Cu} \mathrm{NiTi}$, whereas there is a significant difference between CNA and NiTi wires, for as recieved wires. For used wires, there is a significant difference between TMA wires and the other three types, and there is a significant difference between NiTi, Cu NiTi and CNA. Also, there is a significant difference between NiTi and CuNiTi wires. Three-dimensional images of AFM of all types of wires are seen in Figure 1.

The Pearson correlation coefficient test is shown in Table 4, indicate a significant positive correlation between SR and bacterial adhesion (Figure 2).

TABLE (2) Adhesion of Staphylococcus aureus, Streptococcus mutans and Candida Albicans to the wires.

\begin{tabular}{|c|c|c|c|c|}
\hline & CuNiTi G1 & NiTi G2 & CNA G3 & TMA G4 \\
\hline Staphylococcus aureus & $0.242 \pm 0.019$ & $0.192 \pm 0.035$ & $0.308 \pm 0.122^{\text {b }}$ & $0.376 \pm 0.14^{\text {ab }}$ \\
\hline Streptococcus mutans & $0.220 \pm 0.054$ & $0.174 \pm 0.055$ & $0.337 \pm 0.079^{\text {b }}$ & $0.549 \pm 0.17^{\text {abc }}$ \\
\hline Candida albicans & $0.110 \pm 0.016$ & $0.244 \pm 0.087^{\text {a }}$ & $0.325 \pm 0.038^{\text {ab }}$ & $0.462 \pm 0.054^{\text {abc }}$ \\
\hline
\end{tabular}

a: significance vs. G1 (P<0.05), b: significance vs. G2(P<0.05), c: significance vs. G3(P<0.05) 
TABLE (3) Mean and Standard Deviation (SD) of Surface Roughness of new and used wires.

\begin{tabular}{|c|c|c|c|c|c|c|}
\hline \multicolumn{2}{|c|}{} & CuNiTi G1 & NiTi G2 & CNA G3 & TMA G4 & P value \\
\hline \multirow{3}{*}{ SRa } & New & $63.84 \pm 5.452$ & $54.15 \pm 9.722$ & $74.24 \pm 5.322^{\mathrm{b}}$ & $165.4 \pm 13.45^{\mathrm{abc}}$ & $\mathrm{P}<0.05$ \\
\cline { 2 - 7 } & Used & $107.3 \pm 8.489^{*}$ & $88.61 \pm 9.957^{\mathrm{a}^{*}}$ & $147.5 \pm 8.886^{\mathrm{ab} *}$ & $217.4 \pm 12.59^{\mathrm{abc} *}$ & $\mathrm{P}<0.05$ \\
\hline
\end{tabular}

a: significance vs. G1 (P<0.05), b:significancevs.Group 2(P<0.05),c: significancevs. Group 3(P<0.05), *: significance vs used (within each group) $(P<0.05)$.

Table 4. Pearson correlations between SR and biofilm adhesion to the wires

\begin{tabular}{|l|l|l|l|l|}
\hline & & Staph & Strept & Candida \\
\hline SR & $\mathbf{r}$ & 0.58 & 0.82 & 0.76 \\
\cline { 2 - 5 } & $\mathbf{P}$ & $<0.001^{*}$ & $<0.001^{*}$ & $<0.001^{*}$ \\
\hline
\end{tabular}

r: Pearson's correlation coefficient P: Probability

*: significance $<0.05$

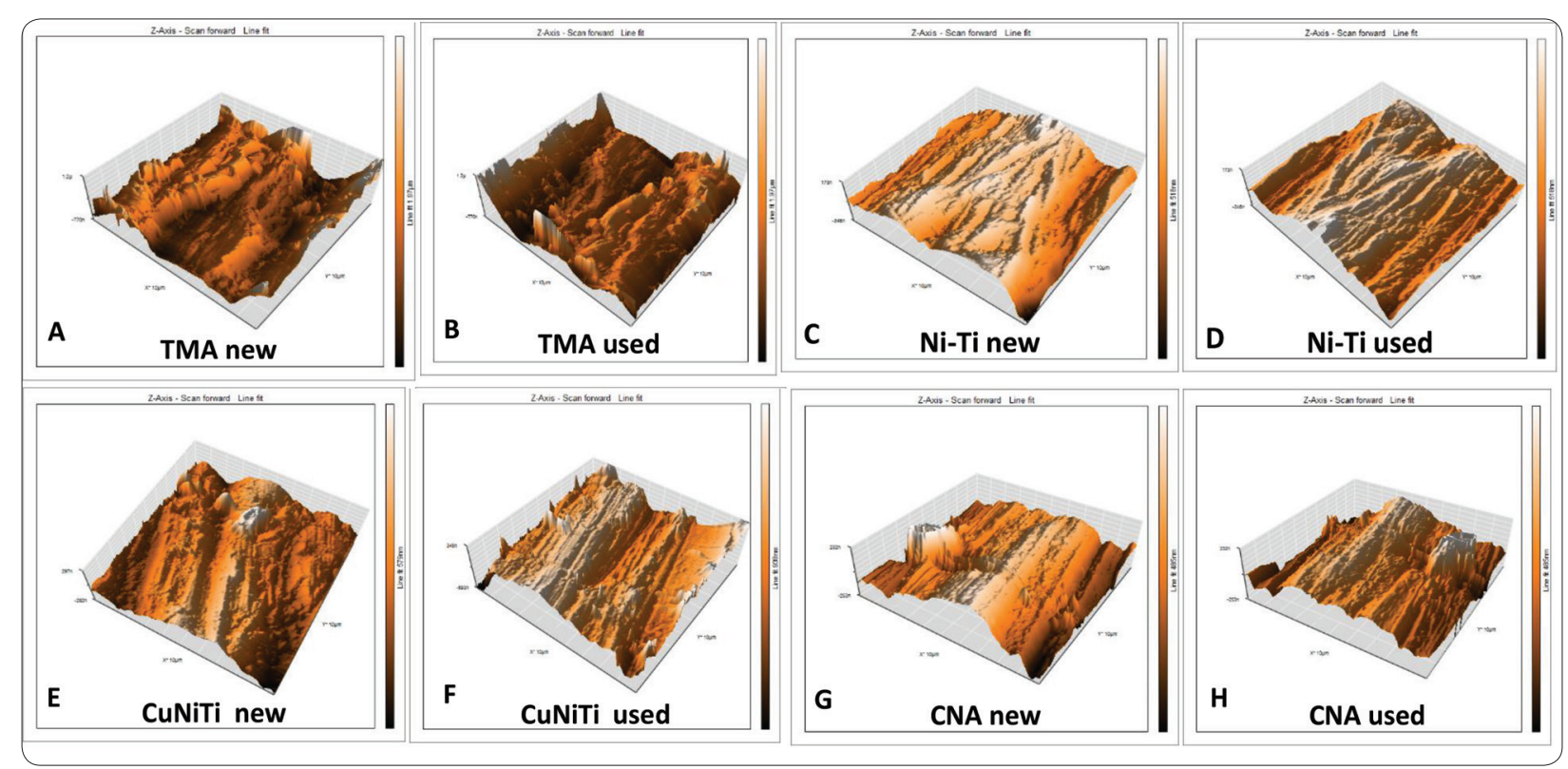

Fig. (1) Three-dimensional atomic force microscope topography images of different new and used wires. 


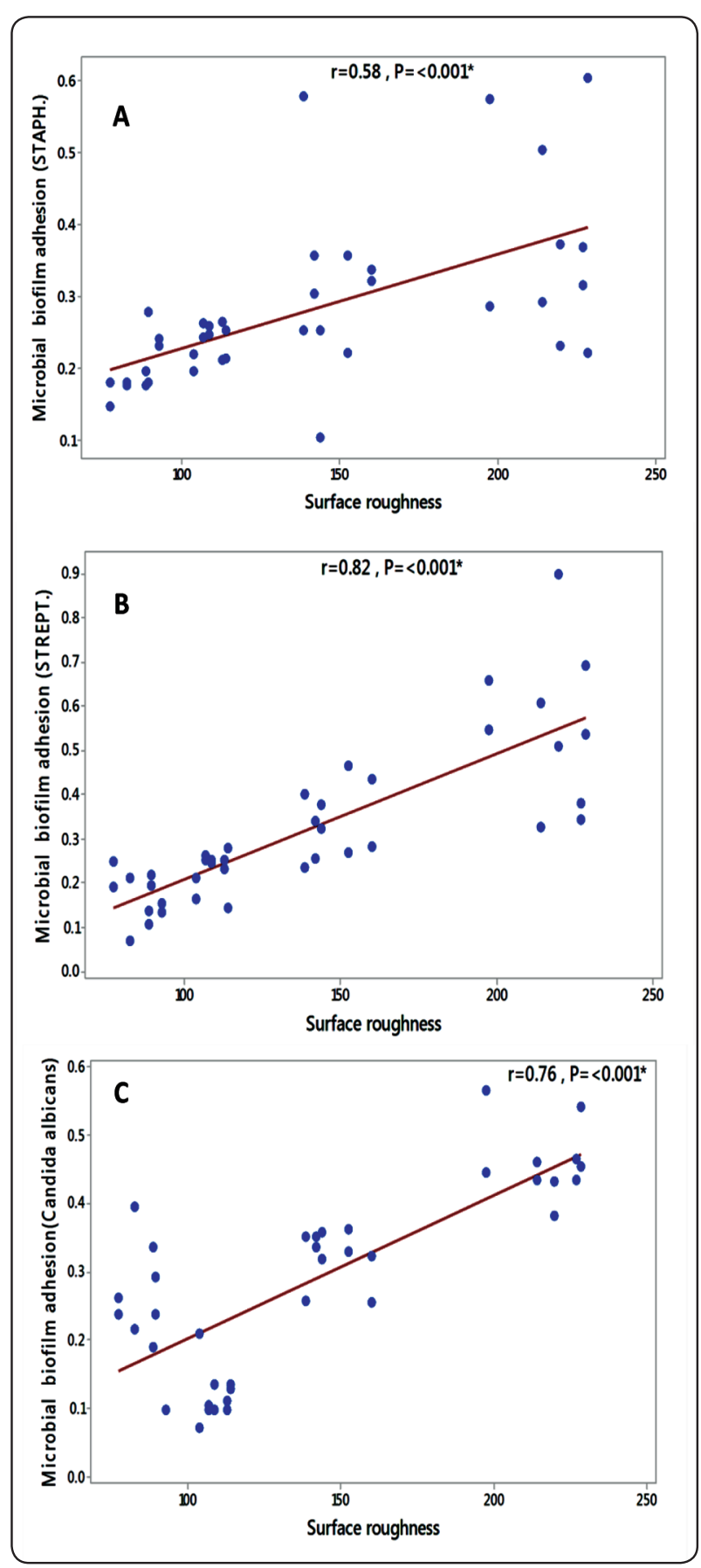

Fig. (2) Pearson correlations between biofilm adherence to the wires and SR of the wire. (A) Staphylococcus aureus, (B) Streptococcus mutans, (C) Candida albicans. $r$ indicates the pearson correlation coeffeicient and $\mathrm{P}$-values for the variables examined.

\section{DISCUSSION}

From the clinical point of view, low surface roughness value of the arch wire leads to fast and easy sliding of the tooth with slight force as the force expended in friction remain at minimal level. A good wire is required to be smooth during the period of its use, but the surface characters of the wire may be affected by the chemical composition of the oral cavity, making it very rough. Consequently, a part of the force which is important to move the tooth is consumed to overcome the frictional force which a major factor for clinical orthodontic success. ${ }^{15}$

In the present study, Atomic force microscopy was used to evaluate the topographic surface characteristics of the arch wires. It is a scanning probe microscope, a sort of tools that, obtain information on the examined surfaces using interatomic interactions, this AFM microscope gains the images by specific sensors, be made up of sharp definite points which intern interact with the specimen surface which is under examination. ${ }^{16-18}$

The results showed significant differences among all new and used wires, NiTi wire showed the lowest roughness values followed by CuNiTi and CNA wires while the highest values either among new or used wires were for TMA. The results agree with previous studies of Doshi and Bhad- Patil, ${ }^{19}$ which showed higher records of surface roughness for TMA, on the other hand they are against the results of others ${ }^{20}$ in which NiTi wires recorded the highest the roughness values. The production technique influences the surface topography of orthodontic wires as the roughness measured for several products from the similar batch was homogeneous. ${ }^{21}$

$\beta$-Ti arch wires (TMA), either new or used ones, recorded the highest roughness values, and this is related to the excessive friction produced by this material.$^{22,23}$ The wire usually displays maximum frictional force, possibly as a result of wear either adhesive or abrasive, which formed with the bracket slot because of the great reactivity with the 
surface of the orthodontic arch wire. ${ }^{24}$ The NiTi wire, in contrast, generates lesser friction than do TMA wire; performance of the arch wire is improved by its stiffness and flexibility. ${ }^{25}$

CNA arch-wires recorded higher surface roughness than NiTi wires with significant difference, and lower than TMA. This finding is in agreement with Juvvadi et al, ${ }^{11}$ as beta-titanium orthodontic arch wires showed a certain degree of surface roughness in SEM examination. This degree of surface roughness, especially at the site of localized cold welding or adherence of the wire and the slot of the bracket, lead to increased arch wire-bracket sliding friction as noted in case of titanium- based archwires. ${ }^{26}$ The CNA wires composed of extra titanium but less molybdenum, zirconium, and tin than TMA wires. Increased strength and hardness of TMA wires is due to increased zirconium, which aids in prevention of embrittling omega phase formation during processing at high temperatures.4 Less zirconium might explain the decreased degree of stiffness of the CNA wires. ${ }^{11,24}$

In the present study, $\mathrm{Cu} \mathrm{NiTi}$ wire showed greater surface roughness than NiTi wires without significant difference for as-recieved types and statistically different for used one, these findings are in agreement with Gravina et al. ${ }^{7}$ and Fischer-Brandies et al. ${ }^{27}$ who showed that $\mathrm{Cu} \mathrm{NiTi} 35^{\circ} \mathrm{C}$ wires had irregular morphological characteristics compared to NiTi wire, these irregularities are drawing marks and slots which be visible under any degree of magnification. Also, it showed microcavities which formed as a result of pull out the particles of $\mathrm{NiTi}$, which could produce a higher coefficient of attrition. In previous studies, it was shown that $\mathrm{Cu}$ NiTi arch-wires have more surface roughness and Streptococcus adhesion than NiTi archwires. ${ }^{15,28}$

Surface roughness values were increased significantly for all types of wires after 4 weeks of intra oral exposure. This finding is related to the abrasive impact of tooth brushing, wearing out due to certain food, and the impinge between the bracket edges and the arch wire, the results of this study were in agreement with the findings of previous studies ${ }^{14,29}$ which evaluated intraoral aging of esthetic coated arch wires, NiTi, and stainless-steel wire, they affirmed that , there is a significant increase in biofilm adhesion and the surface roughness as a result of intraoral exposure of the arch wire.

The surface changes were attributed to the growth of microcrystalline sodium chloride, calcium phosphate, potassium chloride and their accumulation on the wires. These deposits caused pitting and crevice corrosion imperfections along with the decrease of the alloy grain size, as explained in retrieved $\mathrm{NiTi}$ wires in previous studies ${ }^{30,31}$, consequently changing its surface composition and topography. Other important factor for surface changes is the effect of acidogenic bacteria on the surface corrosion behavior of the NiTi wires, thus, changing the surface properties of the used arch wires. ${ }^{32}$

All types of wires showed positive biofilm adhesion after 4 weeks of oral exposure. The highest adhesion values were for TMA wire followed by CNA wire while the lowest values were for NiTi and $\mathrm{CuN}$ iTi wires. The marked increase in surface roughness after oral exposure is the explanation for this result. It was found that the threshold of SR below which it has no influence on bacterial adhesion to the titanium alloy is $0.2 \mu \mathrm{m},,^{33}$ as shown in table 3 , SR values of all types of wires after their usage intraorally were greater than this value. Rough surfaces offer chances for bacterial adherence by increasing the exposed surface area, producing appropriate bacterial adhesion and weakening bacterial colony displacement. ${ }^{34}$ firstly, the bacterial biofilm develops within the valleys of irregular surfaces by permanent adherence of planktonic pioneer bacteria, flattening the coarse parts. ${ }^{35}$ Furthermore, fluctuations in SR of more than $0.1 \mathrm{~mm}$ effect the contact angle, thus altering the surface free energy values, which include the second surface characteristic disturbing bacterial biofilm adhesion to the different orthodontic materials..$^{12,36,37}$ These results are assured by the correlation results, which demonstrate a significant positive correlation between biofilm adhesion and SR of the orthodontic arch wire . 


\section{CONCLUSIONS}

1. Microbial biofilm adhesion was significantly greater in TMA and CNA than NiTi Cu-NiTi arch-wires.

2. The surface roughness of TMA and CNA was greater than NiTi and $\mathrm{Cu}-\mathrm{NiTI}$ arch-wires.

3. Surface roughness for all the examined the archwires in this study increased after 4 weeks of intraoral exposure.

4. There was a positive correlation between surface roughness and the biofilm adhesion on the different wires.

\section{REFERENCES}

1. Filoche, S., Wong, L. And Sissons, C., 2010. Oral biofilms: emerging concepts in microbial ecology. J. Dent. Res., 89:8-18.

2. C.K.HOPE, M. WILSON, Biofilm structure and cell vitality in a laboratory model of subgingival plaque. J Microbiol Methods: 66, 3, pp. 390-398 (2006).

3. Csilla Zambori. Biofilm formation of streptococcus, staphylococcus, pasteurella and neisseria strains Romanian Biotechnological Letters, Vol. 20, No. 4, 2015

4. Diaz, P.I.; Xie, Z.; Sobue, T.; Thompson, A.; Biyikoglu, B.; Ricker, A.; Ikonomou, L.; Dongari- Bagtzoglou, A. Synergistic interaction between Candida albicans and commensal oral streptococci in a novel in vitro mucosal mode Infect. Immun. 2012, 80, 620-632.

5. Cho, T.; Nagao, J.; Imayoshi, R.; Tanaka, Y. Importance of diversity in the oral microbiota including candida species revealed by high-throughput technologies. Int. J. Dent. 2014,454391 .

6. Koo, H.; Bowen, W.H.Candida albicans and Streptococcus mutans A potential synergistic alliance to cause virulent tooth decay in children. Future Microbiol.2014,9, 1295-1297.

7. Gravina MA, Canavarro C, Elias CN, Chaves GM, Brunharo IH, Quinta o CC. Mechanical properties of NiTi and CuNiTi wires used in orthodontic treatment. Part 2: microscopic surface appraisal and metallurgical characteristics. Dent Press J Orthod. 2014;19:69-76.

8. Grass G, Rensing C, Solioz M. Metallic copper as an antimicrobial surface. Appl Environ Microbiol. 2011;77:1541
9. Szuhanek C, Fleser T, Glavan F. Mechanical Behavior of Orthodontic TMA Wires. Issue 3, Wseas transactions on biology and biomedicine 2010;(7):277-286.

10. Alobeid A, Hasan M, Al-Suleiman M, El-Bialy T. Mechanical properties of cobalt-chromium wires compared to stainless steel and $\beta$-titanium wires. J Orthod Sci. 2014 Oct;3(4):137-41.

11. Shubhaker Rao Juvvadi, Vignesh Kailasam, Sridevi Padmanabhan, and Arun B. Chitharanjan. Physical, mechanical and flexutral properties of 3 orthodontic wires: An in vitro study. American Journal of Orthodontics and Dentofacial Orthopedics. 2010;138:623-30.

12. Lee SP, Lee SJ, Lim BS, Ahn SJ. Surface characteristics of orthodontic materials and their effects on adhesion of mutans streptococci. Angle Orthod. 2009;79:353-360.

13. Chun MJ, Shim E, Kho EH, et al. Surface modification of orthodontic wires with photocatalytic titanium oxide for its antiadherent and antibacterial properties. Angle Orthod.2007; 77:483-488.

14. Taha M, El-Fallal A, Degla H. In vitro and in vivo biofilm adhesion to esthetic coated arch wires and its correlation with surface roughness. Angle Orthod. 2016;86:285-291.

15. Yousif AA, Abd El-Karim UM. Microscopic study of surface roughness of four orthodontic arch wires. Tanta Dent J. 2016;13:199-207.

16. Silikas N, Lennie AR, England KER, Watts DC. AFM as a tool in dental research. Microsc Analysis. 2001;82:19-21.

17. Kakaboura A, Fragouli M, Rahiotis C, Silikas N. Evaluation of surface characteristics of dental composites using profilometry, scanning electron, atomic force microscopy and glossmeter. J Mater Sci Mater Med. 2007;18:155-163.

18. Lee GJ, Park KH, Park YG, Park HK. A quantitative AFM analysis of nano-scale surface roughness in various orthodontic brackets. Micron 2010. 41:775-782.

19. Doshi UH, Bhad-Patil WA. Static frictional force and surface roughness of various bracket and wire combinations. Am J Orthod Dentofacial Orthop. 2011;139:74-79.

20. Kusy RP, Whitley JQ, Mayhew MJ, Buckthal JE. Surface roughness of orthodontic archwires via laser spectroscopy. Angle Orthod. 1988;58:33-45.

21. D'Anto V, Rongo R, Ametrano G, et al. Evaluation of surface roughness of orthodontic wires by means of atomic force microscopy. Angle Orthod. 2012;82:922-928. 
22. Kapila S, Sachdeva R. Mechanical properties and clinical applications of orthodontic wires. Am J Orthod Dentofacial Orthop. 1989;96:100-109.

23. Burstone CJ, Goldberg AJ. Beta titanium: a new orthodontic alloy. Am J Orthod. 1980;77:121- 132.

24. Kusy RP, Whitley JQ, de Arau' jo Gurgel J. Comparisons of surface roughnesses and sliding resistances of 6 titanium based or TMA-type archwires. Am J Orthod Dentofacial Orthop. 2004;126:589-603.

25. Matarese G, Nucera R, Militi A, et al. Evaluation of frictional forces during dental alignment: an experimental model with 3 nonleveled brackets. Am J Orthod Dentofacial Orthop.2008;133:708-715.

26. Brantley WA. Orthodontic wires. In: Brantley WA, Eliades T, editors. Orthodontic materials: scientific and clinical aspects. Stuttgart, Germany, and New York: Thieme; 2001. p. $77-103$.

27. Fischer-Brandies H, Es-Souni M, Kock N, Raetzke K, Bock O.Transformation behavior, chemical composition, surface topography and bending properties of five selected $0.016 " \times 0.022 "$ NiTi archwires. J Orofac Orthop 2003; 64:88-99.

28. Gorelick L, Geiger AM, Gwinnett AJ. Incidence of white spot formation after bonding and banding. Am J Orthod. 1982;81:93-98.

29. Rango R, Ametrano G, Gloria A, et al. Effects of intra oral aging on surface properties of surface coated nickel-titanium arch wires. Angle Orthod. 2014;84:665-672.
30. Eliades T, Eliades G, Athanasiou AE, Bradley TG. Surface characterization of retrieved $\mathrm{NiTi}$ orthodontic archwires. Eur J Orthod. 2000;22:317-326.

31. Zegan G, Sodor A, Munteanu C. Surface characteristics of retrieved coated and nickel titanium orthodontic archwires. Rom J Morphol Embryol. 2012;53:935-939.

32. Bahije L, Benyahia H, El Hamzaoui S, et al. Behaviour of NiTi in the presence of oral bacteria: corrosion by Streptococcus mutans. Int Orthod. 2011;9:110-119.

33. Bollen CM, Papaioanno W, VanEldere J, Schepers E, Quirynen $M$, Vansteenberghe $D$. The influence of abutment surface roughness on plaque accumulation and peri-implant mucositi. Clin Oral Implants Res. 1996;7:201-211.

34. Proffit WR. Contemporary Orthodontics. St Louis, Mo: Mosby; 2000.

35. Cortizo C, Fernandez Lorenzo M. Evaluation of early stages of oral streptococci biofilm growth by optical microscopy. Effect of antimicrobial agents. Communicating Current Research and Educational Topics and Trends in Applied Microbiology, A. Mendez-Vilas (Ed.), P. 32-40.

36. Busscher HJ, van Pelt AWJ, de Boer P, de Jong HP, Arends J. The effect of surface roughening of polymers on measured contact angles of liquids. Colloids Surf J. 1984;9:319-331.

37. Kim IH, Park HS, Kim YK, Kim KH, Kwon TY. Comparative short-term in vitro analysis of mutans streptococci adhesion on esthetic, nickel-titanium and stainless steel arch wires.Angle Orthod. 2014;84:680-686 